\title{
Predictability vs. Efficiency in the Multicore Era: Fight of Titans or Happy Ever after?
}

\author{
Luca Benini \\ DEIS Università di Bologna \\ Luca . benini@unibo.it
}

In this talk I will give an overview of recent trends in multi-core platforms for embedded computing. The shift toward multicore architectures has been imposed by technology reasons (power consumption and design closure issues in nanometer technology) and not by the "coming of age" of parallel programming models, compilation, analysis and verification environments. Thus, we may be building terascale many-cores architectures that we cannot program efficiently. Even worse, we may not be able to give any guarantees on execution timing, constraints and real time properties of applications. This is a challenge AND an opportunity for the software design and verification community: I will give some views on what is being done, what could be done and what I hope will be done to build efficient and predictable multi-core platforms. 